\title{
Kinetics of binary nucleation: Multiple pathways and the approach to stationarity
}

\author{
G. Shi and J. H. Seinfeld \\ Department of Chemical Engineering, California Institute of Technology, Pasadena, California 91125
}

(Received 7 June 1990; accepted 13 September 1990)

\begin{abstract}
Explicit analytical expressions are obtained for the rate of nucleation over different paths in a binary system. It is shown that anisotropy in reaction rates and anisotropy in the free energy surface can cause nucleation to occur bypassing the saddle point. Homomolecular nucleation is demonstrated to be the natural limit of binary nucleation as the concentration of one component goes to zero. Explicit expressions are also obtained for the time lag of binary nucleation by using the singular perturbation approach. It is shown that the time lag associated with different paths of nucleation is essential in determining the relative importance of different nucleation pathways.
\end{abstract}

\section{INTRODUCTION}

The identification of a preferred nucleation pathway is essential both for definition of the mechanism of nucleation and calculation of the nucleation rate in a binary system. The nucleation pathway in the classical theory of binary nucleation $^{1}$ is found to go across a saddle point in the free energy surface $(G)$ for the formation of a mixed particle. The steepest descent path corresponding to the minimum energy direction on the surface is defined solely in terms of the geometrical properties of the formation free energy surface without consideration of the monomer concentrations in the system. ${ }^{2,3}$ Consequently, the classical theory of binary nucleation based on the steepest descent path is not kinetically consistent in that it does not reduce correctly to one component homogeneous nucleation theory when one component vanishes. ${ }^{2-4}$ Large discrepancy is also found between the predictions of the classical theory and experimental observations when the concentration of one component is significantly different from that of the other component. ${ }^{5}$

Based on a general multicluster coordinate nucleation theory, ${ }^{6}$ Stauffer ${ }^{2}$ has obtained the correct direction of the nucleation path by considering the properties of both the formation free energy surface and of the nucleating components. His result confirmed an earlier suggestion by Stauffer and Kiang. ${ }^{3}$ However a correct expression for the rate of binary nucleation in a system with significantly different concentrations of the two components has not been obtained, ${ }^{7}$ nor has an expression for the rates of nucleation taking different paths and their relative contributions to the total rate of nucleation. Recent attempts along these lines have been made by Trinkaus, ${ }^{7}$ however a consistent theory of nucleation in a binary system, which reduces correctly to the homomolecular nucleation of one component when the other component is made to vanish, has yet to be developed.

To develop such a theory is one goal of the present paper. An additional goal is to study the role of nonstationarity at the initial stage of nucleation in determining the relative importance of different paths.

The present paper is structured as follows. We will first show that the physical picture of binary saddle point nucleation is similar to that of homomolecular nucleation and the singular pertubation approach used in uniary nucleation can be followed to treat the present binary nucleation problem. Using that approach, we will derive the steady state binary nucleation rate in the case of the cluster flux line going over the saddle point and compare the result with those existing. Then an analysis of multiple nucleation pathways will be given. The relative magnitude of the steady state rate of nucleation over different paths in a binary system will be shown to be controlled not only by the anisotropy in the reaction rates but also by the anisotropy in the shape of the free energy surface. In particular, a continuous transition between heteromolecular nucleation and homomolecular nuclation in a binary system will be predicted. Next, using the singular perturbation approach, we will derive an explicit expression for the effective time lag for binary nucleation. It will be shown that the time lag associated with establishing different nucleation paths is essential in determining the relative importance of different stationary nucleation pathways in contributing the total rate of nucleation.

\section{SINGLE NUCLEATION PATHWAY: CLUSTER FLUX LINES GO OVER THE SADDLE POINT}

\section{A. Basic equations}

The basic equation governing the time-dependent cluster number concentration $f\left(g_{a}, g_{b}, t\right)$ may be written as ${ }^{1,2,7}$

$$
\frac{\partial f\left(g_{a}, g_{b}, t\right)}{\partial t}=-\frac{\partial j_{a}}{\partial g_{a}}-\frac{\partial j_{b}}{\partial g_{b}},
$$

where $g_{i}$ is the monomer number in a cluster $(i=a, b)$, and the cluster flux in composition space is given by

$$
j_{a}=-R_{a} f_{0} \frac{\partial\left(f / f_{0}\right)}{\partial g_{a}}, \quad j_{b}=-R_{b} f_{0} \frac{\partial\left(f / f_{0}\right)}{\partial g_{b}},
$$

where the equilibrium cluster size distribution $f_{0}=n_{0} \exp \left[-\beta G\left(g_{a}, g_{b}\right)\right]$ with $n_{0}$ the total monomer number concentration in the system and $G\left(g_{a}, g_{b}\right)$ is the formation free energy of the clusters. ${ }^{2}$ No explicit form of $G$ is needed in treating the kinetics of nucleation in the present work. $\beta=1 / k T$ where $k$ is Boltzmann's constant and $T$ is 
the absolute temperature. The reaction rate $R_{i}\left(g_{a}, g_{b}\right)$ is defined as the collision rate between monomer $i$ and a cluster of composition $\left(g_{a}, g_{b}\right)$. For example, in the case of gas-phase nucleation, the reaction rate $R_{i}$ is proportional to the cluster surface area and the $i$-component monomer number concentration. ${ }^{1,2}$ In diffusion-limited nucleation in a condensed system, $R_{i}$ is proportional to the radius of a cluster and to the $i$ component monomer concentration. ${ }^{7}$ Both cases will be considered although no explicit form of $R_{i}$ need be specified.

\section{B. Saddle-point binary nucleation}

Consider the case of the saddle point nucleation in a binary system in which no single species is supersaturated with respect to its pure state, i.e., the trajectory of cluster flux lines passes only over the saddle point (conditions for saddle point nucleation will be clarified below). A supersaturation with respect to the mixed-component cluster may develop and clusters may grow spontaneously to be larger stable particles after passing over the saddle point. The cluster size at the saddle point is the so-called the critical size. Thus the physical picture of binary saddle point nucleation is similar to that of homomolecular nucleation and the approach for deriving the rate of uniary nucleation has often been followed to derive the expression for binary nucleation. ' For uniary gas-phase nucleation it has been shown ${ }^{8}$ that the classical steady state rate is exact to the leading order in $\epsilon$, a parameter inversely proportional to $\left(\beta G^{*}\right)^{1 / 2}$. Here $G^{*}$ is the formation free energy barrier at the critical point.

The approach of singular pertubation has proved to be useful in addressing a number of problems in homogeneous nucleation theory. ${ }^{8-10}$ It will prove to be similarly useful in attacking binary nucleation problems. To provide a basis for the analytical approach of this paper, we will first derive the rate of binary saddle point nucleation by using that approach and compare the result with the existing ones. ${ }^{1,2,7}$

Following the procedure presented previously, ${ }^{8}$ we will first derive the equations governing cluster number concentration valid in both the outer and inner regions. The inner region is defined as that in the vicinity of the saddle point. It also can be called the critical region in which an unstable cluster becomes a stable cluster by passing over the saddle point. It is expected that the cluster size distribution within this region is far from equilibrium. In contrast, the cluster size distribution in the outer region is close to the equilibrium distribution corresponding to the individual monomer concentrations.

\section{Outer solutions}

Normalizing Eq. (1) by introducing $z=f / f_{0}$, $m=g_{a} / g_{a}^{*}$ and $n=g_{b} / g_{b}^{*}$, and by neglecting terms proportional to $\epsilon_{a}^{2}$ and $\epsilon_{b}^{2}$, where $\epsilon_{a}=\sqrt{R_{a}^{*}} / g_{a}^{*}$ and $\epsilon_{b}=\sqrt{R_{b}^{*}} / g_{b}^{*}$ which are proportional to $\left(g_{a}^{*}\right)^{-1 / 3}$ and $\left(g_{b}^{*}\right)^{-1 / 3}$, respectively, for gas-phase nucleation and $\left(g_{a}^{*}\right)^{-2 / 3}$ and $\left(g_{b}^{*}\right)^{-2 / 3}$, respectively, for nucleation in a condensed system, we obtain

$$
\begin{aligned}
\frac{\partial z}{\partial t}= & -2 \frac{R_{a}}{g_{a}^{*}} \beta\left[G_{a a}+\frac{G_{b b}}{g_{b}^{*}}(n-1)\right] \frac{\partial z}{\partial m} \\
& -2 \frac{R_{b}}{g_{b}^{*}} \beta\left[G_{b b}+\frac{G_{a a}}{g_{a}^{*}}(m-1)\right] \frac{\partial z}{\partial n} .
\end{aligned}
$$

We have used "*" to indicate the value at the saddle point. Also we have used the quadratic expansion for $G^{1,2,6-8}$

$$
\begin{aligned}
G= & G^{*}+G_{a a}\left(g_{a}-g_{a}^{*}\right)^{2}+G_{b b}\left(g_{b}-g_{b}^{*}\right)^{2} \\
& +2 G_{a b}\left(g_{a}-g_{a}^{*}\right)\left(g_{b}-g_{b}^{*}\right),
\end{aligned}
$$

where $\quad G_{a a}=\frac{1}{2} \partial^{2} G / \partial g_{a}^{2}, \quad G_{b b}=\frac{1}{2} \partial^{2} G / \partial g_{b}^{2}, \quad$ and $G_{a b}=\frac{1}{2} \partial^{2} G / \partial g_{a} \partial g_{b}$ at $g_{a}=g_{a}^{*}$ and $g_{b}=g_{b}^{*}$. The second derivatives describe the shape of the saddle point. While this expansion is more valid near the saddle point, the functional form is expected to be also correct in the outer region. Moreover, it will be shown later a more detailed expression for $G$ does not significantly change the result obtained. The above equation valid in the outer region is not easily solved except for the case in which no cross term appears in $G$. We thus have to diagonalize the matrix

$$
\left(\begin{array}{ll}
G_{a a} & G_{a b} \\
G_{b a} & G_{b b}
\end{array}\right)
$$

which can be accomplished by introducing an ordinary rotational transformation. This transformation can be introduced more clearly in solving for the inner solution given below.

\section{Inner solutions}

Introducing the inner variables $X=(m-1) / \epsilon_{a}$, $Y=(n-1) / \epsilon_{b}$, and $Z=z$, we have the following equation valid in the inner region from Eqs. (1) and (2),

$$
\begin{aligned}
\frac{\partial Z}{\partial t}= & \left(1+\frac{X \sqrt{R_{a}^{*}}+\mu Y \sqrt{R_{b}^{*}}}{g^{*}}\right)^{2 / 3} \\
& \times\left(\frac{\partial^{2} Z}{\partial X^{2}}+\frac{\partial^{2} Z}{\partial Y^{2}}+\frac{\partial \ln f_{0}}{\partial X} \frac{\partial Z}{\partial X}\right. \\
& \left.+\frac{\partial \ln f_{0}}{\partial Y} \frac{\partial Z}{\partial Y}+\frac{2}{3} \epsilon_{a} \frac{g_{a}}{g^{*}} \frac{\partial Z}{\partial X}+\frac{2}{3} \epsilon_{b} \frac{g_{b}}{g^{*}} \frac{\partial Z}{\partial Y}\right),
\end{aligned}
$$

where $\mu$ is the ratio of $a$ - and $b$-monomer volumes and $g^{*}=g_{a}^{*}+\mu g_{b}^{*}$. By neglecting terms containing $\epsilon_{i}$ and smaller in Eq. (5) we have

$$
\frac{\partial Z}{\partial t}=\frac{\partial^{2} Z}{\partial X^{2}}+\frac{\partial^{2} Z}{\partial Y^{2}}-\frac{\partial \beta G}{\partial X} \frac{\partial Z}{\partial X}-\frac{\partial \beta G}{\partial Y} \frac{\partial Z}{\partial Y},
$$

and now

$$
\beta G=\beta G *+D_{a a} X^{2}+D_{b b} Y^{2}+2 D_{a b} X Y
$$

with

$$
\begin{aligned}
& D_{a a}=\beta G_{a a} R_{a}^{*}, \\
& D_{b b}=\beta G_{b b} R_{b}^{*},
\end{aligned}
$$

and

$D_{a b}=\beta G_{a b} \sqrt{R_{a}^{*} R_{b}^{*}}$.

By diagonalization we have

$\beta G-\beta G^{*}=-u x^{2}+v y^{2}, \quad u, v>0$. 
The minus and plus signs in Eq. (7) show that the variables $x$ and $y$ are, respectively, unstable and stable. Here

$u=-\frac{1}{2}\left[D_{a a}+D_{b b}-\sqrt{\left(D_{a a}-D_{b b}\right)^{2}+4 D_{a b}^{2}}\right]$

and

$v=\frac{1}{2}\left[D_{a a}+D_{b b}+\sqrt{\left(D_{a a}-D_{b b}\right)^{2}+4 D_{a b}^{2}}\right]$

which are the two eigenvalues of the matrix

$$
D=\left(\begin{array}{ll}
D_{a a} & D_{a b} \\
D_{b a} & D_{b b}
\end{array}\right) \text {. }
$$

The rotational transformation introduced to diagonalize the bilinear form of $G$ to a quadratic form allowing us to determine the stable and unstable variables is

$x=X \cos \alpha+Y \sin \alpha$,

$y=-X \sin \alpha+Y \cos \alpha$,

$\tan \alpha=\frac{1}{2 D_{a b}}\left[D_{b b}-D_{a a}+\sqrt{\left(D_{a a}-D_{b b}\right)^{2}+4 D_{a b}^{2}}\right]$.

In Eq. (7) that the squares of the variables have different signs means that the energy barrier has a saddle character $\left(G_{a a} G_{b b}-G_{a b}<0\right)$. The determination of the stable and the unstable variables enables us to obtain the outer and inner solutions by solving Eqs. (3) and (6). First we address the inner solution. By using the variables $x$ and $y$ to describe the cluster, Eq. (6) becomes

$$
\frac{\partial z}{\partial t}=\frac{\partial^{2} z}{\partial x^{2}}+\frac{\partial^{2} z}{\partial y^{2}}+2 u x \frac{\partial z}{\partial x}-2 v y \frac{\partial z}{\partial y},
$$

which is solved at steady state to give

$$
w(x)=A \int_{0}^{x} \exp \left(-u k^{2}\right) d k+B
$$

with

$$
w(x)=\int_{-\infty}^{+\infty} z(x, y) \exp \left(-v y^{2}\right) d y .
$$

The constants $A$ and $B$ are to be determined by matching the outer and inner solutions. It is noted that Eq. (11) is similar in the form to the inner equation for homomolecular nucleation. ${ }^{9}$

The direction of the nucleation path at the saddle point is given by $\alpha$ which is an angle with respect to the $g_{a}$ axis. The value of the angle reduces to the classical result of Reiss ${ }^{1}$ for isotropic reaction rates, $R_{a}=R_{b}$. Our result for $\alpha$, Eq. (10), is the same as that obtained by Stauffer. ${ }^{2}$ In particular, in the limit of $R_{b}^{*} \ll R_{a}^{*}, \tan \alpha \rightarrow 0, \alpha=0$, thus the nucleation proceeds along the $g_{a}$-axis direction. At the opposite limit, $\alpha=\pi / 2$, the nucleation proceeds in the $g_{b}$-axis direction. Thus the directions of the nucleation path at those limits are kinetically consistent with physically based expectations. A continuous transition between the angle of the binary nucleation and that of the uniary nucleation of the more abundant component (if its concentration is large enough to be supersaturated) is ensured, i.e., the nucleation path changes from passing over the saddle point on the two-dimensional energy surface to a path going over a point on the mountain ridge in the one-dimensional energy curve. The problem now is why previous theories of binary nucleation do not reduce to that of uniary nucleation in a kinetically consistent way even though the direction of the nucleation path exhibits a proper transition? This is one of the questions we will address in the remainder of the paper.

\section{Matching inner and outer solutions}

Using the coordinate rotation introduced above to obtain the inner solution we can obtain the left steady state outer solution from Eq. (3),

$$
z_{\text {left }}=1
$$

This left outer solution satisfies one of the boundary conditions (B.C.) to Eq. (1), i.e.,

$$
z \rightarrow 1 \text { as } g_{a}+g_{b} \rightarrow 1 .
$$

Satisfying the remaining B.C.,

$$
z \rightarrow 0 \text { as } g_{a}+g_{b} \rightarrow \infty,
$$

the right outer solution is given by

$$
z_{\text {right }}=0 \text {. }
$$

Matching the inner solution with the left outer solution,

$$
\lim _{x \rightarrow-\infty} \lim _{\epsilon_{i} \rightarrow 0} \lim _{x \rightarrow 1} z=\lim _{x \rightarrow-\infty} \lim _{\epsilon_{i} \rightarrow 0} \lim _{x \rightarrow 1} z_{\text {left }}
$$

and the right outer solution

$$
\lim _{z \rightarrow \infty} \lim _{\epsilon_{i} \rightarrow 0} \lim _{X \rightarrow 1} z=\lim _{x \rightarrow \infty} \lim _{\epsilon_{i} \rightarrow 0} \lim _{X \rightarrow 1} z_{\text {right }}
$$

gives

$$
A=\frac{u}{\sqrt{R_{a}^{*} R_{b}^{*}|\operatorname{det} G|}},
$$

and

$$
B=\frac{1}{2} \sqrt{\pi / v}
$$

with $\operatorname{det} G=G_{a b}^{2}-G_{a a} G_{b b}$

\section{Steady-state rate of binary saddle-point nucleation}

The total cluster flux in the size space in the case of binary nucleation is difficult to define.$^{1,2,6,7}$ From Eqs. (11) and (12), we have

$$
\frac{\partial w}{\partial t}=\frac{\partial^{2} w}{\partial x^{2}}+2 u \frac{\partial w}{\partial x}=-\frac{1}{f_{0}} \frac{\partial}{\partial x}\left(-f_{0} \frac{\partial w}{\partial x}\right)
$$

or

$$
\frac{\partial}{\partial t} \int_{-\infty}^{+\infty} f(x, y) d y=-\frac{1}{f_{0}} e^{v y^{2}} \frac{\partial}{\partial x}\left(-f_{0} \frac{\partial w}{\partial x}\right) .
$$

The Jacobian of the transformation going from variables $g_{a}, g_{b}$ to $x, y$ in $f$ is $\sqrt{R_{a}^{*} R_{b}^{*}}$. Changing the variables $x, y$ into $g_{a}, g_{b}$ in $f$ on the left-hand side of Eq. (17), we have

$$
\begin{aligned}
& \frac{\partial}{\partial t} \int_{-\infty}^{+\infty} f\left[x\left(g_{a}, g_{b}\right), y\left(g_{a}, g_{b}\right)\right] d y\left(g_{a}, g_{b}\right) \\
& \quad=-\frac{\partial}{\partial x}\left(-\sqrt{R_{a}^{*} R_{b}^{*}} f_{0} e^{v y^{2}} \frac{\partial w}{\partial x}\right) \equiv-\frac{\partial}{\partial x} J,
\end{aligned}
$$


where $J$ is the one-dimensional cluster flux along the unstable variable $x$, averaged over the stability variable $y$

$J=-\sqrt{R_{a}^{*} R_{b}^{*}} f_{0} e^{v y^{2}} \frac{\partial w}{\partial x}=-\sqrt{R_{a}^{*} R_{b}^{*}} \int_{-\infty}^{+\infty} f_{0} \frac{\partial z}{\partial x} d y$.

The left-hand side of Eq. (18) is the rate change of the cluster number concentration which is now equivalent to the left-hand side of Eq. (1); thus $J$ defined by Eq. (18) is also the total flux of clusters in the composition space. The rate of nucleation is given by the total cluster flux at the critical size, and we finally get for the steady-state rate of binary nucleation

$J_{s s}^{*}=-\sqrt{R_{a}^{*} R_{b}^{*}} f_{0} e^{v y^{2}} \frac{\partial w}{\partial x}=\frac{u}{\beta \sqrt{\operatorname{det} G}} n_{0} e^{-\beta G^{*}}$.

The above expression for the rate of binary nucleation is similar in form to that of uniary nucleation (which is given by $Z R^{*} f_{0}^{*}$, where $Z$ is the Zeldovich factor, $R{ }^{*}$ is the reaction rate of a critical-sized cluster, and $f_{0}^{*}$ is the cluster number concentration at the critical size). An important difference lies in the fact that the reaction rate in the expression for uniary nucleation is replaced by a complicated factor $u$ that is determined by the reaction rates as well as by the shape of the free energy surface at the saddle opint [Eq. (8)]. Our result for the steady-state rate of binary nucleation with the cluster flux lines passing over the saddle point is exactly the same as that obtained by Trinkaus ${ }^{7}$ who employed a different coordinate transformation. Our result is also the same as that obtained by Stauffer. ${ }^{2}$

\section{Limiting behavior of saddle point binary nucleation}

The limiting behavior of binary nucleation taking a saddle trajectory for significantly different reaction rates is sought to examine the kinetic consistency of the theory.

Considering $R_{b} \ll R_{a}$ (we can also of course consider the analogous limit, $R_{a} \ll R_{b}$ ) we have from Eq. (8),

a. $G_{a a}<0$. Negative $G_{a a}$ at the saddle point implies that a barrier exists along the axis $g_{a}$ on $G$ for a given small concentration of $g_{b}$. In this case, from Eq. (8),

$$
u \approx \beta R_{a}^{*}\left|G_{a a}\right| \text {. }
$$

Thus, the kinetic factor in the rate of binary nucleation [Eq. (20) ] is controlled by the fast (or more abundant) component $a$ (i.e., $R_{a} \gg R_{b}$ ), which seems on the surface to be contradictory to physical intuition and the direction of the nucleation path. This inconsistency has been attributed to the assumption that the trajectory of the cluster flux line even in the limit of $R_{a} \gg R_{b}$ crosses the saddle point ${ }^{7,11,12}$ However, we will show later that the steady-state prediction by Eq. (20) with $u$ given by Eq. (21) is kinetically unattainable because an infinite time lag is required as $R_{a}^{*} / R_{b}^{*} \rightarrow 0$ for $G_{a a}<0$. Thus in this case the total rate of nucleation is zero because neither component is supersaturated. Therefore, the kinetic consistency of binary nucleation theory is ensured for the case of $R_{a}^{*} \gg R_{b}^{*}$ and $G_{a a}<0$ which will be further discussed below. When the $a$ component is supersaturated with respect to its pure state, the total rate of nucleation in the system equals the rate of nucleation of the $a$ component at a given small concentration of the $b$ component with a path along a ridge of the one-dimensional free energy curve. A kinetically consistent theory of nucleation has to be developed by considering multiple nucleation pathways as will be done in the next section.

b. $G_{a a}>0$. Positive $G_{a a}$ means the saddle point is a minimum along the $g_{a}$-axis in $G$ for a given negligible concentration of $g_{b}$. Under this condition,

$$
u \approx \beta R_{b}^{*} \operatorname{det} G / G_{a a} .
$$

Thus, the slow (or the less abundant) component controls the kinetic factor in the nucleation rate. In the limit of $R_{b}^{*} \rightarrow 0$, the rate of binary nucleation thus goes to zero as expected physically. In this case as also shown below the steady-state rate predicted here [Eqs. (20) and (22)] might be realized without constraints due to the long time lag as for the case of $R_{a a}<0$.

c. $G_{a a}=0 . G_{a a}=0$ implies that the saddle point is not a critical point along the $g_{a}$ axis for a given small concentration of $g_{b}$. In this case, from Eq. (8),

$$
u \approx \frac{1}{2} \beta G_{a b} \sqrt{R_{a}^{*} R_{b}^{*}}
$$

and the kinetic factor in the nucleation rate is determined by both the slow and fast components. In the limit of $R_{b}^{*} \rightarrow 0$, the rate of binary nucleation thus goes to zero as expected physically. As shown in a section below this is the most ideal case of a saddle point nucleation whether or not $a$ is supersaturated with respect to its pure state.

In the above three cases when $R_{b} \ll R_{a}$, the cluster flux line first traces along the equilibrium line $\partial G / \partial g_{a}=0$, unless it either reaches the coordinate $g_{b}^{*}$ of a saddle point with $G_{a a}<0$, or it reaches, after having passed over a saddle point with $G_{a a}>0$, a point $G_{a a}=0$ of unstable growth with respect to component $a$, or it just reaches the saddle point for the case of $G_{a a}=0$. At those points it bends over into the fast reacting direction $a$ [Eq. (10)]. It should be noted that similar discussions are given for the first two cases by Trinkaus. ${ }^{7}$

In the following we will explore the relative contributions of different nucleation pathways to the total rate of nucleation (binary and uniary) in the system.

\section{MULTIPLE NUCLEATION PATHWAYS: CLUSTER FLUX LINE BYPASSES THE SADDLE POINT}

As discussed above when one reaction rate is significantly different from the other the flux line can turn into the direction of the fast-reacting component before the saddle point coordinate of the slowly reacting component is reached. If the fast-reacting component is supersaturated with respect to its pure state, the cluster line can pass over the ridge in the direction of $a$ before $g_{b}^{*}$ is reached. This ridge pathway can always coexist with the saddle-point pathway since for very large values of $g_{a}$ and fixed $g_{b}$ the formation free energy approaches $-\infty$ for the pure bulk liquid of $a$ 
component. The valley pathway and the ridge pathway are two solutions to $\partial G / \partial g_{a}=0$ for a given small $g_{b}$ when the activity of $a$ is greater than $1 .^{3}$

The possibilities of multiple nucleation pathways were discussed by Stauffer and Kiang ${ }^{3}$ but no results were derived. A recent attempt ${ }^{7}$ to deal with this problem quantitatively was not successful. The problem lies in using an incorrect governing equation and an incorrect definition for the rate of nucleation as discussed below.

It is known physically that in the case of significantly different reaction modes, the fast mode adjusts itself to the slow one, the slow-reacting one thus determines the overall kinetics of the process. ${ }^{13}$ For a binary nucleation system with significantly different reaction rates, the rate of overall nucleation is governed by the slow component. This means that we can exclude the rapidly relaxing variable, reducing the governing equation to an effective one-dimensional equation. This can be done by using the method of elimination of the fast variable. ${ }^{13}$

The resulting one-dimensional effective equation, obtained by integrating Eq. (1) with respect to $g_{a}$, is

$$
\frac{\partial J\left(g_{b}\right)}{\partial g_{b}}+j_{a}=-\frac{\partial \int f d g_{a}}{\partial t}
$$

where the total cluster flux is

$$
J=\int j_{b} d g_{a} .
$$

$j_{a}$ is the cluster flux bending from the equilibrium line (or socalled valley defined by $\partial G / \partial g_{a}=0$ ), passing over the ridge,

$$
j_{a}\left(g_{b}\right)=\left[\frac{\beta\left|G_{a a}^{r}\right|}{2 \pi}\right]^{1 / 2} R_{a}^{r} n_{0} \exp \left(-\beta G^{r}\right),
$$

where $r$ indicates values on the ridge defined by $\partial G / \partial g_{a}=0$, $\partial^{2} G / \partial g_{a}^{2}<0$. Thus by averaging over the fast variable in the anisotropic limit, the two-dimensional barrier crossing problem is reduced to a one-dimensional barrier crossing problem. The governing equation [Eqs. (24) to (26)] is new. Eq. (24) describes the cluster number conservation (continuity).

It is noted that $j_{a}$ given by Eq. (26) is different from the expression for the same flux given in previous work. ${ }^{7}$ They used $f$ for $n_{0}$ in Eq. (25) resulting in an incorrect governing equation which is similar in the form to the diffusion equation with a sink term. ${ }^{14,15}$ The governing equation obtained is also similar to the governing equation for homomolecular nucleation in the presence of cluster depletion. ${ }^{8-10}$

At steady state, by using Eq. (26), Eq. (24) can be integrated to give

$$
J_{s s}^{r}=\left[\frac{\beta\left|G_{a a}^{r}\right|}{2 \pi}\right]^{1 / 2} R_{a}^{r} n_{0} \exp \left(-\beta G^{\mathrm{eff}}\right),
$$

where

$$
\exp \left(-\beta G^{\mathrm{eff}}\right)=\int_{-\infty}^{+\infty} \exp \left(-\beta G^{r}\right) d g_{a} .
$$

We see that the binary nucleation is reduced to a uniary nucleation with an effective free energy $G^{\text {eff }}\left(g_{b}\right) . G^{\text {eff }}\left(g_{b}\right)$ is essentially equal to $G^{\text {eff }}\left[g_{a}\left(g_{b}\right), g_{b}\right]$, where $g_{a}\left(g_{b}\right)$ is the value of $g_{a}$ in the valley defined by $\partial G / \partial g_{a}=0$. This valley defines the minimum energy path leading from the outer region through the saddle point to a stable-growth region. In general, we can obtain the rate of nucleation passing over the ridge of the one-dimensional curve $G{ }^{\text {eff }}$ from Eq. (27) which is one of the important results obtained. However, depending on the relative magnitudes of $g_{b}^{r}$ and $g_{b}^{*}$, we can determine the relative contributions of different nucleation pathways to the total rate of nucleation (binary and uniary) from Eq. (27). (Here $g_{b}^{r}$ and $g_{b}^{*}$ are the $b$-monomer numbers in the two critical sizes existing in a binary system with the activity of component $a$ greater than 1. One is the usual saddle point; the other, the activation size, is the one at which point the barrier to addition of molecules of the supersaturated component vanishes.)

\section{A. $g_{b} \approx g_{b}^{*}$}

In the saddle point region the expansion for $G$ given by Eq. (4) can be used. From Eq. (27), the steady-state rate of nucleation at $g_{b}=g_{b}^{r} \approx g_{b}^{*}$ and $g_{a}=g_{a}^{r} \approx g_{a}^{*}$ is given by

$$
J_{s s}^{r}=\left[\frac{-G_{a a}^{r}}{G_{b b}}\right]^{1 / 2} R_{a}^{r} n_{0} \exp \left(-\beta G^{*}\right) .
$$

The ratio between $J_{s s}^{r}$ and the rate of the saddle-point nucleation in the limit of $R_{a} \gg R_{b}$ with $G_{a a}^{r} \leqslant 0$ is given by

$$
\frac{J_{s s}^{*}}{J_{s s}^{r}}=\frac{1}{\sqrt{\left|1-\left(G_{a b}^{2} /\left|G_{a a} G_{b b}\right|\right)\right|}} .
$$

Thus, the relative contributions of the saddle point path and the mountain ridge path depend on the anisotropy in the free energy surface at the saddle point. When

$$
1>G_{a b}^{2}>\left|G_{a a} G_{b b}\right|
$$

or

$$
1>\left|G_{a a} G_{b b}\right|>G_{a b}^{2},
$$

then $J_{s s}^{*}>J_{s s}^{r}$, i.e., the contribution to the rate of nucleation from the saddle point is more important than that from the ridge path. For a symmetrical potential surface, $G_{a b}^{2}=\left|G_{a a} G_{b b}\right|, J_{s s}^{*} / J_{s s}^{r} \rightarrow \infty$. Also, $J_{s s}^{r}=0$ for $G_{a a}=0$. In those cases, the cluster flux lines can only pass over the saddle point regardless if either of the single species is supersaturated with respect to its pure state because of the dominant importance of the free energy surface. In other words under those conditions only saddle-point nucleation can occur. Of course in the limit of $R_{b}^{*} \rightarrow 0$, the rate of saddle point nucleation also tends to zero as given by Eqs. (20) and (23).

When the free energy surface at the saddle point becomes very anisotropic such that the ratios between $G_{a b}^{2}$ and the product $G_{a a} G_{b b}$ become larger than 1, then the cluster flux line can bypass the saddle point completely and the mountain ridge nucleation path becomes dominant in contributing to the total rate of nucleation. This is typically the case for a flatter potential surface.

Equation (29) is also valid for isotropic reaction rates, 
i.e., $R_{a}=R_{b}$. Thus we have shown that aniostropic reaction rates are not the only cause for cluster flux lines to avoid passing over the saddle point. This saddle point avoidance caused by anisotropy in the potential surface near the saddle point even for isotropic reaction rates might also be a general phenomena in multidimensional activated rate process. ${ }^{16-18}$

\section{B. $g_{b}^{\prime} \gg g_{b}^{*}$}

Two pathways can be followed by the cluster flux line. The first one is the single barrier path. It is the usual path along which the cluster flux line will first go across the saddle point remain in the valley. Clusters may grow without thermodynamic constraints by accretion of both $a$ and $b$ monomers. This saddle point trajectory is the minimum resistance pathway. Another possible pathway is a double barrier pathway. After passing over the saddle point, clusters may avoid the valley and jump the mountain ridge at $g^{r}$ to grow by adding mostly $a$ monomers.

Since the rate of this double barrier nucleation is smaller than that of the single barrier saddle-point nucleation by a factor of $\exp \left(-G^{r}\right)$ one expects

$$
\frac{J^{r}}{J^{s p}} \approx 0
$$

and the contribution from the saddle point path is the decisive one in determining the total nucleation rate in a binary system when $g_{b}^{r} \gg g_{b}^{*}$.

\section{C. $g_{b}^{*} \ll g_{b}^{*}$}

In this case we can expand $G^{r}$ about $g_{b}^{r}=0$,

$$
\boldsymbol{G}^{r}=\boldsymbol{G}_{g b=0}^{r}+G_{b}^{r} g_{b}+G_{a}^{r}\left(g_{a}-g_{a}^{r}\right),
$$

where $G_{b}=\partial G / \partial g_{b}, G_{a}=\partial G / \partial g_{a}$ at $g_{b}=g_{b}^{r}=0$, and $g_{a}=g_{a}^{r}$. Thus, at $g_{a}=g_{a}^{r}$ and $g_{b}=0$,

$$
\begin{aligned}
J_{s s}^{r} & =-\int j_{a} d g_{b} \\
& =\left(\frac{\beta\left|G_{a a}^{r}\right|}{2 \pi}\right)^{1 / 2} R_{a}^{r} n_{0} \frac{\exp \left(-\beta G_{g_{b}=0}\right)}{\beta G_{b}^{r}} \\
& =\left(\frac{\beta\left|G_{a a}^{r}\right|}{2 \pi}\right)^{1 / 2} R_{a}^{r} n_{0} \exp \left(-\beta G^{\dagger}\right),
\end{aligned}
$$

where

$$
G^{\dagger}=G_{g_{b}=0}^{r}-\frac{1}{\beta} \ln \left(\beta G_{b}^{r}\right) .
$$

Since

$$
\frac{1}{\beta} \ln \left(\beta G_{b}^{r}\right)
$$

is negligibly small in comparison with $G_{g_{b}=0}^{r}$ we have

$$
G^{\dagger} \approx G_{g_{b}=0}^{r} \text {. }
$$

Equation (31) thus gives the exact expression for the rate of a uniary nucleation of component $a$.

We have therefore demonstrated for the first time a continuous transition between binary nucleation and uniary nu- cleation and the conditions under which this transition occurs.

The contribution to the total nucleation rate from nucleation across the saddle point is given by Eq. (20), with the eigenvalue given by Eq. (21). The ratio of the rate of saddle nucleation and to that of the ridge nucleation is

$$
\begin{aligned}
J_{s s}^{*} / J_{s s}^{r}= & \frac{R_{a}^{*}}{R_{a}^{r}} \frac{\left|G_{a a}\right|}{\sqrt{|\operatorname{det} G|}}\left(\frac{2 \pi}{\beta\left|G_{a a}^{r}\right|}\right)^{1 / 2} \\
& \times \exp \left[-\beta\left(G^{*}-G^{r}\right)\right],
\end{aligned}
$$

which is smaller than 1 if $G^{*} \gg G^{r}$ but it is larger than 1 if $\operatorname{det} G$ goes to zero. This is in contrast with our expectation that $J_{s s}^{r} \gg J_{s s}^{*}$. Also it is surprising to find that the ratio does not depend on the ratio of $R_{a} / R_{b}$. That the ratio of the steady-state rate of nucleation taking the saddle point path over that taking the ridge path does not depend on the ratio of the reaction rates is also in contrast with previous propositions $^{3}$ and conclusions. ${ }^{7}$

It will be shown in the next section that the steady-state assumption of different paths might not be realized. The time lag associated with the different paths controls the relative importance in contributing to the total rate of nucleation in a binary system, and it is indeed the ratio of the reaction rates that determines the time lag. Thus a consistent theory for nucleation in a binary system must include the effect of this initial nonstationarity.

Before going on, it is worthwhile pointing out that in previous studies, ${ }^{7}$ the governing equation used was incorrect as mentioned above, and the cluster flux at $g_{b}=0$ was used as the rate of nucleation, which is only correct for $g_{b}^{r} \ll g_{b}^{*}$.

\section{TRANSIENT KINETICS OF BINARY NUCLEATION}

\section{A. Effective time lag in binary nucleation}

The pathways of nucleation that have been discussed up to now are stationary ones. We have shown that binary nucleation reduces to uniary nucleation of the fast-reacting component $a$ when $R_{a} \gg R_{b}$ and $G_{a a}<0$. But what if no single species is saturated with respect to its pure state when $R_{a} \gg R_{b}$ ? Physically neither binary nucleation nor uniary nucleation should exist. The prediction given by Eqs. (20) and $(21)$ is in contrast with this physically based expectation. In the following we will show the steady-state rate of binary nucleation as predicted by Eqs. (20) and (21) is kinetically unattainable because of the associated infinitely long time lag.

An explicit expression for the time lag for binary nucleation in the case of the trajectory of the cluster flux line passing over the saddle point will be obtained by solving the timedependent governing equation (1) using the singular perturbation approach.

\section{Outer solution}

Equation (1) can be simplified as follows:

$$
\frac{\partial z}{\partial t}=-R_{a} \frac{\partial z}{\partial g_{a}} \frac{\partial \beta G}{\partial g_{a}}-R_{b} \frac{\partial z}{\partial g_{b}} \frac{\partial \beta G}{\partial g_{b}}
$$


in the outer region as shown in Sec. II.

Using the coordinate rotation adopted above, we have

$\frac{\partial z}{\partial t}=-2 u x(1+c x)^{2 / 3} \frac{\partial z}{\partial x}-2 v y(1+c x)^{2 / 3} \frac{\partial z}{\partial y}$,

where

$$
c=\frac{\sqrt{R_{a}^{*}} \cos \alpha+\mu \sqrt{R_{b}^{*}} \sin \alpha}{g^{*}} .
$$

Solving Eq. (33) by introducing $z(x, y, s)=\int_{0}^{\infty} \exp (-s t) z(x, y, t) d t$, we obtain the Laplace transformation of the outer solution as

$$
\begin{aligned}
z_{\text {left }}= & \frac{1}{s}\left(\frac{1-\xi}{1-\xi_{0}}\right)^{s / 2 u}\left(\frac{1+\xi+\xi^{2}}{1+\xi_{0}+\xi_{0}^{2}}\right)^{-s / 4 u} \\
& \times \exp \left\{-\sqrt{3} \frac{s}{2 u}\left[\tan ^{-1}\left(\frac{2 \xi+1}{\sqrt{3}}\right)\right.\right. \\
& \left.\left.-\tan ^{-1}\left(\frac{2 \xi_{0}+1}{\sqrt{3}}\right)\right]\right\}
\end{aligned}
$$

where

$$
\xi=(1+c x)^{1 / 3}
$$

and $\xi_{0}$ is the value of $\xi$ at $g_{a}=1, g_{b}=1$.

For the diffusion-limited nucleation in a condensed phase system, we have

$$
\begin{aligned}
z_{\text {left }}= & \frac{1}{s}\left(\frac{1-\xi}{1-\xi_{0}}\right)^{s / 2 u}\left(\frac{1+\xi+\xi^{2}}{1+\xi_{0}+\xi_{0}^{2}}\right)^{-s / 4 u} \\
& \times \exp \left\{\sqrt { 3 } \frac { s } { 2 u } \left[\tan ^{-1}\left(\frac{2 \xi+1}{\sqrt{3}}\right)\right.\right. \\
& \left.\left.-\tan ^{-1}\left(\frac{2 \xi_{0}+1}{\sqrt{3}}\right)\right]\right\}
\end{aligned}
$$

and the right outer solution still is $z_{\text {right }}=0$ for both cases.

In obtaining the above outer solutions we have assumed that the solutions depend only on the unstable variable $(x)$. The reason that cluster nucleation can be generally described in terms of a single nucleation path in the outer region is that for relative large $g^{*}$ the system is essentially in equilibrium everywhere outside the saddle point region. In the inner (saddle point) region the solution has to be obtained by solving the complete equation in terms of unstable and stable variables as will be done below. The accuracy of our solution will be confirmed by reducing it to the known asymptotic result. Our solution is an improvement over that of Wilemski ${ }^{19}$ who assumed that the solution depends solely on the unstable variable over the whole region, which is physically incorrect as he pointed out. Another improvement is that we have adopted a more proper coordinate transformation scheme. Wilemski assumed that $\tan \alpha=g_{b}^{*} / g_{a}^{*}$ which is also incorrect physically in view of the present result [Eq. (10) ]. Finally our result is analytically expressed in terms of the properties of the free energy surface and the reaction rates of the two species present. A stochastic approach ${ }^{11}$ and a variational method ${ }^{12}$ have been developed to study the transient kinetics of binary nucleation, but no explicit analytical expression for the time lag was obtained.

\section{Inner solution}

Solving the time-dependent equation (11) by using Laplace transformation, we obtain the inner solution in terms of two repeated error functions,

$$
w=A i^{\mathrm{s} / 2 u} \operatorname{erfc}(x \sqrt{u})+B i^{\mathrm{s} / 2 u} \operatorname{erfc}(-x \sqrt{u}),
$$

which is the same for both gas-phase and diffusion-limited condensed phase nucleation.

\section{Matching outer and inner solutions}

By matching the outer and inner solutions we get

$$
\begin{aligned}
A= & \frac{1}{2 s} \Gamma\left(1+\frac{s}{2 u}\right)\left\{\frac{c \sqrt{1+\xi_{0}+\xi_{0}^{2}}}{3 \sqrt{3 u}\left(1-\xi_{0}\right)}\right. \\
& \left.\times \exp \left[-\sqrt{3}\left(\frac{\pi}{3}-\tan ^{-1} \frac{1+2 \xi_{0}}{\sqrt{3}}\right)\right]\right\}^{s / 2 u} \sqrt{\frac{\pi}{v}}
\end{aligned}
$$

for the case of the gas phase, and

$$
\begin{aligned}
A= & \frac{1}{2 s} \Gamma\left(1+\frac{s}{2 u}\right)\left\{\frac{c \sqrt{1+\xi_{0}+\xi_{0}^{2}}}{3 \sqrt{3 u}\left(1-\xi_{0}\right)}\right. \\
& \left.\times \exp \left[\sqrt{3}\left(\frac{\pi}{3}-\tan ^{-1} \frac{1+2 \xi_{0}}{\sqrt{3}}\right)\right]\right\}^{s / 2 u} \sqrt{\frac{\pi}{v}}
\end{aligned}
$$

for the case of diffusion-limited nucleation in the condensed phase, and $B=0$ for both cases.

\section{Time-dependent rate of binary nucleation}

After an inversion, we have, from Eqs. (38)-(40),

$$
w=\frac{1}{2} \sqrt{\frac{\pi}{v}} \operatorname{erfc}\left[\sqrt{u} x+\exp \left(-\frac{t-\lambda \tau}{\tau}\right)\right]
$$

for both of the diffusion-limited and gas-phase nucleation. Using Eq. (19) and the above obtained cluster size distribution,

$$
J=J_{s s}^{*} \exp \left[-\exp \left(-2 \frac{t-\lambda \tau}{\tau}\right)\right]
$$

where

$$
\tau=(2 u)^{-1}
$$

is the characteristic time scale of binary nucleation determined by the collision frequency between monomers and a critical-sized mixed cluster, and

$$
\begin{aligned}
\lambda= & \sqrt{3}\left(\frac{\pi}{3}-\tan ^{-1} \frac{1+2 \xi_{0}}{\sqrt{3}}\right) \\
& +\ln \left[\frac{3 \sqrt{3 u}\left(1-\xi_{0}\right)}{c \sqrt{1+\xi_{0}+\xi_{0}^{2}}}\right]
\end{aligned}
$$

for gas-phase nucleation. For diffusion-limited condensed phase nucleation, we have,

$$
\begin{aligned}
\lambda= & -\sqrt{3}\left(\frac{\pi}{3}-\tan ^{-1} \frac{1+2 \xi_{0}}{\sqrt{3}}\right) \\
& +\ln \left[\frac{3 \sqrt{3 u}\left(1-\xi_{0}\right)}{c \sqrt{1+\xi_{0}+\xi_{0}^{2}}}\right]
\end{aligned}
$$


and $c$ is given by Eq. (34). The difference between the gasphase and diffusion-limited cases is obviously due to the difference in the reaction rates.

Equation (42) is given at $g_{a}=g_{a}^{*}$ and $g_{b}=g_{b}^{*}$. Different time dependency can be observed at different cluster sizes. It is also noted that the time dependency of the rate of binary of nucleation is similar to that of uniary nucleation. ${ }^{9}$ This double exponential dependency of the transient rate of nucleation differs functionally from all other previously obtained results (for a more detailed discussion see Ref. 9).

\section{Effective time lag of binary nucleation}

The number density of critical sized clusters formed in the system following the increase in activities of both components at $t=0$ is the integrated flux $N(t)=\int_{0}^{t} J\left(t^{\prime}\right) d t^{\prime}$. Using Eq. (42), the dimensionless total number is

$$
\begin{aligned}
\frac{N(t)}{J_{s s} \tau} & =\frac{1}{2} \int_{e^{-2(1 / \tau-\lambda)}}^{e^{2 \lambda}} \frac{d x}{x e^{x}} \\
& =\frac{1}{2}\left[E_{1}\left(e^{-2(t / \tau-\lambda)}\right)-E_{1}\left(e^{2 \lambda}\right)\right],
\end{aligned}
$$

where $E_{1}$ is the exponential integral.

As $(t / \tau-\lambda) \rightarrow \infty$, we have $e^{-2(t / \tau-\lambda)} \rightarrow 0$, and $E_{1}\left[e^{-2(t / \tau-\lambda)}\right]=-\gamma-2(\lambda-t / \tau)+O\left[e^{-2(t / \tau-\lambda)}\right]$, thus

$$
\frac{N(t)}{J_{s s} \tau}=-\frac{1}{2}\left[E_{1}\left(e^{2 \lambda}\right)+\gamma+2 \lambda\right]+\frac{t}{\tau},
$$

where $\gamma=0.5772$ is Euler's constant.

Eq. (47) thus indicates that at large times, $N(t)$ increases linearly with time $t$ as expected. The beginning of the steady state regime can be determined experimentally by the time at which the increase of $N(t)$ becomes linear with time. The time lag $\left(\tau_{e}\right)$ defined in such a way is the effective time lag which is given by

$$
\tau_{e}=\frac{1}{2}\left[E_{1}\left(e^{2 \lambda}\right)+\gamma+2 \lambda\right] \tau
$$

with $\lambda$ given by Eq. (44) for gas-phase nucleation and Eq. (45) for diffusion-limited nucleation in the condensed phase.

The effective time lag for binary nucleation is functionally the same as that for uniary nucleation. ${ }^{9}$ In analogy to uniary nucleation, the term $c^{-1} \sqrt{u}$ is equivalent to the small parameter $(\epsilon)$. Also the present expression for $\tau$ reduces to the same $\tau$ in the case of an uniary nucleation when $R_{a}$ or $R_{b}$ equals zero. Thus we can examine the accuracy of the quadratic expansion for $G$ in solving for the time lag for the binary nucleation by comparing the expressions for the time lag for uniary nucleation using the quadratic expansion for $G$ and using the complete conventional expression for $G$. In using the quadratic expression,

$$
\begin{aligned}
\lambda= & \sqrt{3}\left[\frac{\pi}{3}-\tan ^{-1} \frac{2\left(g^{*}\right)^{-1 / 3}+1}{\sqrt{3}}\right] \\
& +\frac{1}{2} \ln \left[\frac{3}{1+\left(g^{*}\right)^{-1 / 3}+\left(g^{*}\right)^{-2 / 3}}\right]
\end{aligned}
$$

$$
+\ln \left\{\frac{3}{\epsilon}\left[1-\left(g^{*}\right)^{-1 / 3}\right]\right\}
$$

while

$$
\lambda=\left(g^{*}\right)^{-1 / 3}-1+\ln \left\{\frac{3}{\epsilon}\left[1-\left(g^{*}\right)^{-1 / 3}\right]\right\}
$$

using the complete conventional expression for $G .{ }^{9}$ For $g^{*}=30$, the difference between the two expressions for $\lambda$ is $1.55 \tau$. Using the quadratic approximation, we have overestimated the value of $\lambda$. Since the term $\ln (3 / \epsilon)$ is the dominant one in the expression for the $\lambda$, the small difference $(1.55 \tau)$ in the above expressions for $\lambda$ implies that the quadratic expansion for $G$ used in the present work for deriving the expression for the time lag of binary nucleation is acceptable. However if $G$ is known the procedure presented can be followed to obtain a more accurate expression as done in the case of an uniary nucleation. ${ }^{9}$

\section{B. Transient kinetics and nucleation paths in a binary system}

Let us first examine the time lag at the limit of $R_{b} \ll R_{a}$. In this limit, we have

$$
\begin{aligned}
& c^{-1} \sqrt{u} \approx \frac{g^{*}}{\mu} \sqrt{\frac{\beta R_{a}^{*}\left|G_{a a}\right|}{R_{b}^{*}}}, \quad G_{a a}<0, \\
& c^{-1} \sqrt{u} \approx \frac{g^{*}}{\mu} \sqrt{\frac{\beta|\operatorname{det} G|}{G_{a a}}}, \quad G_{a a}>0, \\
& c^{-1} \sqrt{u} \approx \frac{g^{*}}{\mu} \sqrt{\frac{\beta G_{a b}}{2} \sqrt{\frac{R_{a}^{*}}{R_{b}^{*}}}}, \quad G_{a a}=0 .
\end{aligned}
$$

Thus, we observe the following important behavior. First for $G_{a a}<0$, the time lag for saddle point nucleation in the limit of $R_{b} \ll R_{a}$ can be relatively long (depending on the ratio of $\left.R_{a}^{*} / R_{b}^{*}\right)$ since $\tau_{e}$ is proportional to $\ln \left(3 c^{-1} u\right)$. As $R_{b} / R_{a}$ goes to zero, the time lag $\tau_{e}$ approaches infinity. The physics of the problem is clear. If the concentration of one species becomes vanishingly small, heteromolecular nucleation becomes impossible when the remaining species is not supersaturated with respect to its pure state. An infinite time lag of nucleation corresponds to a zero rate of binary nucleation in this case. Thus, although Eqs. (20) and (21) predict that the steady-state rate of nucleation for $G_{a a}<0$ depends on the fast reactive component $a$, the actual rate depends on the transient kinetics. If $a$ is supersaturated with respect to its pure state, then for $R_{a} \gg R_{b}$ and $G_{a a}<0$, since the time lag of heteromolecular nucleation is longer than that for ridge nucleation, the degeneration of the saddle-point binary nucleation into uniary nucleation as discussed in Sec. III (case 3) will be realizable.

We have thus resolved the conflicit ${ }^{7}$ between the prediction by Eqs. (20) and (21) in the case of $R_{a} \gg R_{b}$ and $G_{a a}<0$ and the physical expectation. The theory of nucleation in a binary system is therefore consistently developed.

The first case $\left(G_{a a}<0\right)$ shows how the anisotropy in the reaction rates affects the transient kinetics of nucleation. The second case $\left(G_{a a}>0\right)$ will show how the anisotropy in 
the free energy surface affects the transient kinetics. From the above expression for $G_{a a}>0$, as $|\operatorname{det} G|$ decreases, the time lag of nucleation increases. It should be noted that in this case the time lag is independent of the anisotropy in the reaction rates. Although the transient kinetics might not be the controlling factor in realizing the steady-state rate of nucleation, the rate is controlled by the slow-reacting component $b$ as predicted by Eqs. (20) and (23). If $a$ is also supersaturated with respect to its pure state, then the concept of nucleation becomes meaningless for $G_{a a}^{\text {eff }}>0$ at the ridge ( $G_{a a}^{\text {eff }}>0$ because of $G_{a a}>0$ ).

In the third case of $G_{a a}=0$, the effective time lag also increases with increasing $R_{a} / R_{b}$. In this case the ridge crossing nucleation is impossible as discussed in Sec. III A. The prediction for the rate of saddle point nucleation given by Eqs. (20) and (23) will be realized after a relatively long time lag for a nonzero concentration of $b$.

\section{RELATIONSHIP TO OTHER ACTIVATED RATE PROCESSES}

Kramers classical theory of activated barrier crossing deals only with the case of isotropic diffusion rates. Recent$l y$, the role of anisotropy in both potential surface and diffusion rates in affecting the rate of barrier crossing has been an active area of investigation. ${ }^{16-18,20-22}$ Since nucleation is an activated barrier crossing rate process, our present results have some implications to the other activated barrier crossing processes. First our results show that saddle-point avoidance can occur even for isotropic diffusion rates when the shape of the potential surface in the saddle region is very asymmetric. In the context of chemical kinetics, this effect is still under study. ${ }^{19,23}$

The saddle point avoidance caused by the anisotropy in diffusion rates is related to the time lag required for system to reach its steady state. The importance of transient kinetics in determining the rate of multidimensional barrier crossing process has not been fully addressed. ${ }^{21}$ It will be interesting to apply the present procedure to study the rate and the transient kinetics of a general multidimensional barrier crossing process.

\section{CONCLUSIONS}

Explicit analytical expressions are obtained for the rate of nucleation taking different stationary paths in a binary system. It is shown that not only the anisotropy in reaction rates but also the anisotropy in the free energy surface can cause nucleation to bypass the saddle point completely. Homomolecular nucleation is shown to be the natural limit of heteromolecular nucleation as the concentration of one component goes to zero. Explicit expressions are also obtained for the time lag of binary nucleation by using the singular perturbation approach. Knowledge of the steady state rate of nucleation taking different paths is not sufficient to determine the relative importance of different paths. The saddle point avoidance caused by the anisotropy in diffusion rates is shown to be related to the time lag required for system to reach its steady state.

\section{ACKNOWLEDGMENT}

This work was supported by National Science Foundation Grant No. ATM-9003186.

'H. Reiss, J. Chem. Phys. 18, 840 (1950).

${ }^{2}$ D. Stauffer, J. Aerosol Sci. 7, 319 (1976).

${ }^{3}$ D. Stauffer and C. S. Kiang, Icarcus 21, 129 (1974).

${ }^{4}$ P. Mirabel and J. L. Clavelin, J. Aerosol Sci. 9, 219 (1978).

${ }^{5}$ P. Mirabel and J. L. Katz, J. Chem. Phys. 60, 1138 (1973); 67, 1138 (1977); J. L. Schmitt, J. Whitten, G. W. Adams and R. A. Zalabsky, J. Chem. Phys. 92, 3693 (1990); P. Wagner and G. Vali, Lecture Notes in Physics, Vol. 309, Atmospheric Aerosols and Nucleation (Springer, Berlin, 1988), pp. 3 and 367; S. M. Kreidenweis, R. C. Flagan, J. H. Seinfeld and K. Okuyama, J. Aerosol Sci. 20, 585 (1989).

${ }^{6} K$. Binder and D. Stauffer, Adv. Phys. 25, 343 (1976)

${ }^{7}$ H. Trinkaus, Phys. Rev. B 12, 7372 (1983).

${ }^{8} \mathrm{G}$. Shi and J. H. Seinfeld, J. Colloid Interface Sci. 135, 252 (1990).

${ }^{9}$ G. Shi, J. H. Seinfeld and K. Okuyama, Phys. Rev. A 41, 2101 (1990).

${ }^{10} \mathrm{G}$. Shi and J. H. Seinfeld, J. Chem. Phys. 92,687 (1990).

"'W. J. Shugard and H. Reiss, J. Chem. Phys. 65, 2827 (1976).

${ }^{12}$ W. F. J. Schelling and H. Reiss, J. Chem. Phys. 74, 3527 (1981).

${ }^{13} \mathrm{C}$. W. Garder, Handbook of Stochastic Methods for Physics, Chemistry and Natural Sciences (Springer, Berlin, 1985).

${ }^{14}$ N. Agmon and J. J. Hopfield, J. Chem. Phys. 78, 6947 (1983).

${ }^{15}$ H. Sumi and R. A. Marcus, J. Chem. Phys. 84, 4894 (1986).

${ }^{16}$ A. M. Berezhkovskii and V. Y. Zitserman, Chem. Phys. Lett. 158, 369 (1989).

17A. M. Berezhkovskii, L. M. Berezhkovskii, and V. Y. Zitserman, Chem. Phys. 130, 55 (1989).

${ }^{18}$ R. S. Larson and M. D. Kostin, J. Chem. Phys. 77, 149 (1983).

${ }^{19} \mathrm{G}$. Wilemskii, J. Chem. Phys. 62, 3772 (1975).

${ }^{20}$ R. S. Larson, Physica A 137, 295 (1986).

${ }^{21}$ M. M. Klosek-Dygas, B. M. Hoffman, B. J. Matkowsky, A. Nitzan, M. A. Ratner, and Z. Schuss, J. Chem. Phys. 90, 1141 (1989).

${ }^{22}$ M. Berkowitz, J. D. Morgan, J. A. McCammon, and S. H. Northrup, J. Chem. Phys. 79, 5563 (1983)

${ }^{23}$ B. J. Matkowsky, A. Nitzan and Z. Schuss, J. Chem. Phys. 88, 4765 (1988); 90, 1292 (1989); and R. S. Larson, ibid. 90, 1291 (1989). 